\title{
Optimal Network Topology Based on Natural Connectivity and Improved Genetic Algorithm
}

\author{
Lei Ming ${ }^{1, a}$, Guoqiang Yan ${ }^{2, b}$ \\ ${ }^{1}$ Science and Technology on Information Systems Engineering Laboratory, Nanjing, 210007, China \\ ${ }^{2}$ Science and Technology on Information Systems Engineering Laboratory, Nanjing, 210007, China \\ aemail: storm_sky001@126.com, b email: dennis.gqyan@foxmail.com
}

Keywords: Natural Connectivity; Genetic Algorithm; Cloud Mode

\begin{abstract}
Invulnerability study is an important research content of complex network. On the basis of analysis and comparison of other traditional inadequate measurements of invulnerability, natural connectivity was used as the invulnerability measurement of network topology and an optimal network topology model based on natural connectivity has been proposed. Based on the basic idea of genetic algorithms, we designed an improved algorithm based on genetic algorithm and cloud mode to solve the model. Simulation experiments analyzed the invulnerability changes before and after the network topology optimization and verified the rationality of network topology optimization model and algorithm.
\end{abstract}

\section{Introduction}

An important purpose of complex network research is to design a good network topology and invulnerability is an important measurement to check the network. Currently, the study of complex network invulnerability is based on graph theory. The graph theory based invulnerability indicators mainly include toughness [1], integrity [2], tenacity [3], algebraic connectivity [4][5], etc., but these indicators have their disadvantages [6][7]. Chvatal initially used tenacity to study Hamiltonicity a graph. Later, it was used to measure the graph invulnerability, but Bauer et al demonstrated that the calculation of toughness was a NP problem. The integrity does not only consider the degree of difficulty of network destruction, but also takes into account of the scale of the largest piece after being destroyed, but the calculation of integrity is also a NP problem. Similarly, tenacity does not solely focus on the degree of difficulty of network destruction, but also takes into account of the scale of the maximum connectivity piece scale and the number of connectivity pieces, but the calculation of tenacity is also a NP problem. Algebraic connectivity is obviously inadequate, and not suitable for large-scale networks. Nature connectivity proposed by Literature [8] focused on proceeding from the internal network topology properties to describe the redundancy of alternative path by the method of closed path amount weighing, with clear physical significance and in concise mathematical form. It can be directly obtained by calculating the characteristic spectrum of the adjacent matrix, with a relatively low calculation complexity. Moreover, the edge adding and removal of natural connectivity is in strict monotonic increase or decrease, which has obvious advantages by comparing with other indicators and can well measure the invulnerability of network topology. Therefore, this paper uses natural connectivity as the invulnerability measurement to establish network topology optimization model, design the algorithm based on genetic algorithm and cloud model, which does not only speed up the search speed of the algorithm, but also reduces the possibility of local optimum. 


\section{Network Topology Invulnerability Optimization Model}

\section{Network topology representation.}

Complex network topology can be represented as the Figure $G=(V, E)$ composed of node set $V$ and edge set $E$, where $V$ is a node set, represented as $V=\left\{v_{1}, v_{2}, \cdots, v_{N}\right\}$, edge set represented as $E=\left\{e_{1}, e_{2}, \cdots, e_{W}\right\} \subseteq V \times V$, indicating node connectivity. $N=|V|$ is used to represent node quantity, whereas $W=|E|$ represents edge quantity, $E(G)=\left(a_{i j}\right)_{N \times N}$ means the adjacent matrix of $G$, where $a_{i j}$ represents the connection between network node $i$ and $j$ :

$$
a_{i j}=\left\{\begin{array}{l}
1 \quad i \text { and } j \text { is connected } \\
0 \quad \text { otherwise }
\end{array}\right.
$$

$\bar{\lambda}$ is the natural connectivity of Figure $G$, where

$$
\bar{\lambda}=\ln \left(\frac{1}{N} \sum_{i=1}^{N} e^{\lambda_{i}}\right)
$$

Where ${ }^{\lambda_{i}}$ is the characteristic root of the adjacent matrix $E(G)$.

\section{Network topology optimization model.}

Natural connectivity is used as the network invulnerability to optimize the network topology, while assume network $G$ as the undirected graph, that is, $a_{i j}=a_{j i}, a_{i i}=0$. Assume the network is an unweighted graph, that is

$$
a_{i j}= \begin{cases}1 & \left(v_{i}, v_{j}\right) \in E \\ 0 & \left(v_{i}, v_{j}\right) \notin E\end{cases}
$$

At the same time, assume the network is a connected graph, that is, $\mu_{N-1}>0$, where $\mu_{N-1}$ is the second smallest characteristic root of Laplace matrix, namely, algebraic connectivity.

Network natural connectivity strictly increases with the number of edges, but the network construction has cost limitation. The greater number of edge is, the higher cost of the network construction is. Therefore, we use the number of medium edges as the constraint conditions to research how to maximize the network invulnerability under the constraints of a fixed value. According to the aforementioned analysis, complex network optimization model can be set up:

$$
\begin{aligned}
& \max \quad \bar{\lambda}(G)=\ln \left(\frac{1}{N} \sum_{i=1}^{N} e^{\lambda_{i}}\right) \\
& \left\{\begin{array}{l}
\sum_{i=1}^{N} \sum_{i=1}^{N} a_{i j}=2 W \\
a_{i j}=a_{j i} \\
a_{i i}=0 \\
\mu_{N-1}>0 \\
a_{i j}=1 \text { or } 0
\end{array}\right.
\end{aligned}
$$

\section{Model Solution Based on Genetic Algorithm and Cloud Model}

Genetic Algorithms (GA) is a random search algorithm [9][10] proposed by Professor John Holland of Michigan University by referring to biological natural selection and natural genetic mechanism in 1975. Unlike traditional optimization algorithm, genetic algorithm has no specific 
model requirements. For example, the target function is not required to be continuous and differentiable; its search process depends solely on the fitness function, which has simple operation, with strong adaptability for solving a variety of problems. Network optimization is an issue of combinatorial optimization, and genetic algorithm has great advantage. Currently, genetic algorithm has made some achievement in the research of combinatorial optimization. In theory, genetic algorithm has the capability to find the optimum solution, but there are some deficiencies. Standard genetic algorithm uses fixed crossover and mutation probability, but it is prone to prematurity, slow convergence and other problem. Moreover, genetic parameters set for specific issues also require a lot of experiments, without a universal method. Compared with genetic algorithm, the adaptive genetic algorithm proposed by literature [11] has made significant improvement in improving the convergence speed and maintain population diversity. However, when the algorithm is at the highest fitness degree, the genetic operator tends to 0 , increasing the possibility of falling into prematurity [12]. Therefore, some scholars proposed to set the lower limit to the genetic parameters, but most of the abovementioned methods are improved on the basis of linear function or by constructing specific functions, which cannot reflect self-adaption of the parameter, with obvious weakness. Therefore, we need to take full advantage of genetic algorithm while overcome its shortcomings; at the same time, for different problems and research fields, we shall also propose the corresponding methods and strategies by combining with their own characteristics, thereby further improving the search efficiency and quality of genetic algorithms.

We know the concept value described by natural language is more accurate and universal than mathematical expression. However, how to express the uncertainty in natural language concepts, especially the randomness and fuzziness? To solve these problems, academician Li Deyi started from fuzzy mathematics and probability theory to study the cloud model, namely, the uncertainty transformation model between a certain qualitative concept and the quantified value, providing a powerful processing means to the qualitative and quantitative conversion. After nearly 20 years' improvement and development, cloud model has been successfully applied in algorithm improvement, data mining, comprehensive evaluation, knowledge representation and other fields [13][14]. By referring to the idea of genetic algorithm, this paper uses cloud model to design crossover operator and mutation operator to improve genetic operation, proposing an algorithm based on genetic algorithm and cloud model. The approach can both improve the search efficiency of the algorithm and also reduce the possibility of running into local optimum.

\section{Profile of Cloud Model.}

\section{1) Cloud model}

Suppose $U$ is a quantitative domain of discourse (one-dimensional, two-dimensional or multidimensional) represented by a precise number and $C$ is a qualitative concept of domain $U$. For any element $x$ in $U$, there is a random number $\mu(x)$

$$
\mu: U \rightarrow[0,1], \forall x \in U, x \rightarrow \mu(x)
$$

$\in[0,1]$ with stable tendency

Mapping of qualitative concept $C$ from domain of discourse $U$ to interval [0,1], in other word, the distribution in discourse domain $U$ is known as cloud model $C$, simply referred to as cloud [13][14]. Each $x$ is called a cloud droplet, $\mu(x)$ is called the certainty of $x$ towards $C$. The randomly realized quantitative values $x$ represents the randomness of concept quantitative value, whereas the certainty $\mu(\mathrm{x})$ of $x$ for concept $C$ reflects the certainty degree of numerical $x$ representing qualitative concept. Cloud model, better reflecting the uncertainty of natural concept, is the transformation model of uncertainty between qualitative knowledge and quantitative representation.

The cloud uses three numerical characteristics for the representation of a concept, namely, expectation Ex, entropy En and hyper entropy He [13][14].

Expectation $E x$ is the expectation of cloud droplets in discourse domain spatial distribution, which is the point best representing this qualitative concept, the most typical sample point of this concept quantization, indicating the position of cloud's gravity core.

Entropy En is the uncertain measurement of qualitative concept, reflecting the randomness and fuzziness of the concept. On one hand, En reflects the cloud droplets' degree of dispersion representing the qualitative concept; on the other hand, it reflects the cloud droplet valuing range 
acceptable to qualitative concept.

Hyper entropy $\mathrm{He}$ is the uncertain measurement of En, namely the entropy of entropy, which is jointly determined by randomness and fuzziness of En, indicating the cloud 'thickness' in terms of cloud shape.

The 3 numerical characteristic values of cloud model fully integrate the fuzziness and randomness by a specific construction algorithm to form the conversion model between qualitative concept and quantitative representation. Cloud model no longer emphasizes precise function representation, but uses three numerical characteristics to show the concept uncertainty, and then realize the uncertainty conversion of qualitative concept and quantitative representation, while revealing the relevance between fuzziness and randomness [13][14]. On one hand, cloud model can convert the quantitative knowledge into a quantitative characterization, which generates cloud droplet by numerical characteristics, known as forward cloud generator; on the other hand, cloud model can produce qualitative concept via quantitative data, that is, to get the cloud model numerical characteristics by cloud droplet distribution, known as backward cloud generator.

\section{2) Normal Cloud}

Normal cloud model is one of the cloud models with universal adaptability. Suppose $U$ is a quantitative domain represented by a precise number and $C$ is a qualitative concept of domain $U$. If any quantitative value $x$ is the one-time random achievement of qualitative concept $C$. If $x$ satisfies $x \sim N\left(E x, E n^{2}\right)$, where $E n^{\prime} \sim \mathrm{N}\left(E n, H e^{2}\right)$ and the certainty of $x$ towards $\mathrm{C}$ satisfies:

$$
\mu=e^{-\frac{(x-E x)^{2}}{2(E n)^{2}}}
$$

The distribution of $x$ in the domain of discourse $U$ is known as normal cloud [13][14].

\section{3) Forward normal cloud generator}

The algorithm of forward normal cloud generator $G(E x, E n, H e)$ is as follows [13][14]:

(1) Generate a normal random number $E n^{\prime}=\operatorname{Norm}\left(E n, H e^{2}\right)$ with $E n$ as the expectation and $H e^{2}$ as variance

(2) Generate a normal random number $x=\operatorname{Norm}\left(E x, E n{ }^{\prime 2}\right)$ with $E x$ as the expectation and $E n{ }^{\prime 2}$ as variance;

(3) Calculate $\mu=e^{-\frac{(x-E x)^{2}}{2 E n^{2}}}$

(4) $x$ with certainty $\mu$ become a cloud droplet of the digital domain.

(5) Repeat step (1) to (4) until generating the n cloud droplet as required.

Given $E X 1=10, E n 1=1, H e 1=0.1$ and $E X 2=10, E n 2=5, H e 2=0.5$ and $\mathrm{n}=500$, joint distribution $C(10,1,0.1)$ and $C(10,5,0.5)$ are generated respectively, whose one-dimensional normal cloud figure is shown in Figure 1. The concept expressed by Figure 1 is "in the vicinity of 10" to carry out quantitative representation of the qualitative concept of entropy and hyper entropy. Cloud droplet in Figure 1 can be understood as expectation of 10, the 2 populations with the number of individuals of 500 obtained by different entropy and hyper entropy. The two populations have significant difference in coverage range and discretion degree, the greater the entropy is, the wider the individual coverage is; the greater the hyper entropy is, the more discrete the individuals are. The three numerical characteristics, Ex, En, He, of cloud model are used to represent the genetic operators. He, in particular, can well represent the evolutionary stable tendency and randomness. The smaller He means weaker uncertainty, whereas greater He means stronger uncertainty. 


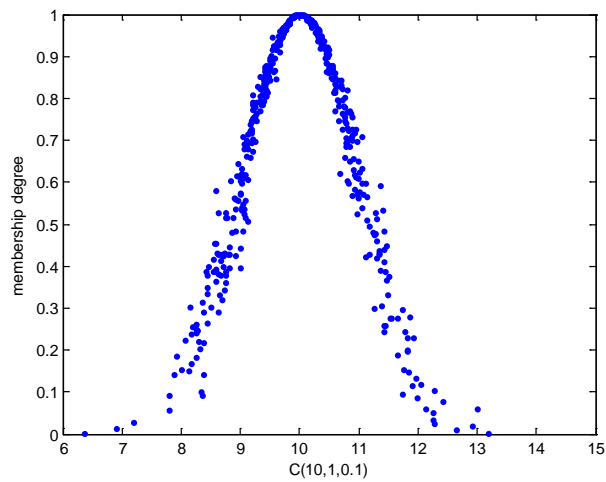

(a) Cloud Model $C(10,1,0.1)$

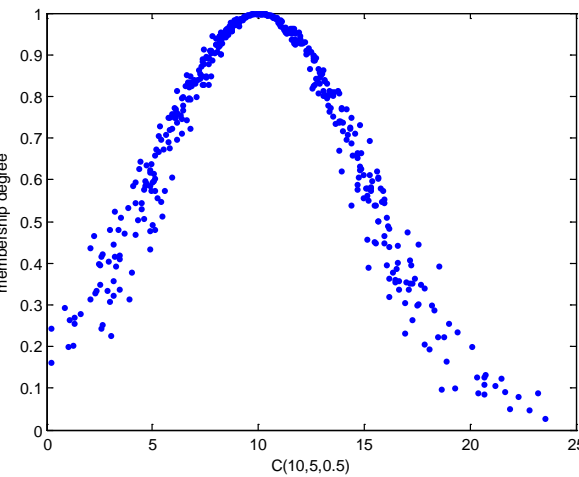

(b) Cloud Model $C(10,5,0.5)$

Fig.1. Concept of "in the Vicinity of 10" Represented by Cloud Model

\section{Solution process based on genetic algorithm and cloud model}

\section{1) Selection operation}

In the basic genetic algorithm, the probability of individual being copied depends on its fitness value. Because of random operation, the phenomenon of worst individual loss may occur in the search process. To protect the diversity of the population, we set the protection probability Ppro, the individuals with minimum fitness will be entailed to the next generation according to the probability Ppro.

\section{2) Crossover operation}

This paper avails the good characteristics of cloud model's randomness and fuzziness and uses the cloud model to purchase crossover and mutation probability to make the probability value in good trend, at fast convergence speed, with strong randomness while reduce the possibility of algorithm in local optimum. The crossover probability algorithm based on cloud model is as follows:

$$
\begin{aligned}
& E X=\frac{f_{1}+f_{2}}{2} \\
& E n=l_{1}\left(f_{\max }-f^{\prime}\right) \\
& H e=l_{2} E n \\
& E n=\operatorname{Norm}(E n, H e) \\
& P_{c}= \begin{cases}k_{1} e^{\frac{-\left(f^{\prime}-E x\right)^{2}}{2(E n)^{2}}}, f^{\prime} \geq \bar{f} \\
k_{2}, & f^{\prime}<\bar{f}\end{cases}
\end{aligned}
$$

Where, $P_{c}$ represents crossover probability; $f_{\max }$ is the population maximum fitness values. $f_{1}$ and $f_{2}$ are the two crossed individuals' fitness values. $f^{\prime}$ is the greater value of the two individual fitness. $l_{1} 、 l_{2} 、 k_{1} 、 k_{2}$ are control parameters, where $l_{1} \in[0.4,0.6], l_{2} \in[0.3,0.8], k_{1}, k_{2} \in[0,1]$. This paper uses $l_{1}=l_{2}=0.4, \quad k_{1}=k_{2}=1$.

\section{3) Mutation operation}

The cloud model based mutation probability algorithm is as follows:

$$
\begin{aligned}
& E X=f \\
& E n=l_{3}\left(f_{\max }-f\right) \\
& H e=l_{4} E n \\
& E n^{\prime}=\operatorname{Norm}(E n, H e) \\
& P_{m}= \begin{cases}k_{3} e^{\frac{-(f-E x)^{2}}{2(E n)^{2}}}, f \geq \bar{f} \\
k_{4}, & f<\bar{f}\end{cases}
\end{aligned}
$$

Where, $P_{m}^{\prime}$ represents mutation probability; $f_{\max }$ is maximum fitness value of the population 
and $f^{\prime}$ is the mutation individual fitness value. $l_{3}, l_{4}, k_{3}, k_{4}$ are control parameters, where $l_{3} \in[0.05,0.2] 、 l_{4} \in[0.05,0.25] 、 k_{3}, k_{4} \in[0,1]$. This paper uses $l_{3}=l_{4}=0.1, k_{1}=k_{2}=0.5$.

Thus, for individuals whose fitness is lower than the average fitness of the population, maximum crossover and mutation probability is used to produce superior individuals. At the same time, for individuals higher than population average fitness, the greater the fitness is, the smaller the crossover mutation probability is, but is not 0 , so as to "protect" optimum individuals.

\section{4) Algorithm running termination conditions}

We set the maximum number of iteration first. When the individual fitness value in the maximum number of iterations has no change or little change, we can determine the population convergence and the iterative process ends, otherwise iteration will continue. After the suspension of the iteration, individuals with the highest degree of fitness value are selected as the optimal solution, and network topology optimization will be obtained by decoding.

\section{Simulation experiment}

\section{Basic parameter setting.}

In the simulation process, ER network of complex network model is selected as the research object. Take the number of nodes $N=30$, number of edges $W=35$ and probability of edges' connection $P=0.08$, the generated ER graph is shown in Figure 3 and the natural connectivity is 1.0034. In the process of solving network topology optimization model, the relevant parameter setting is: population size of 300 , the maximum genetic times of 500 and $P p r o=0.3$.

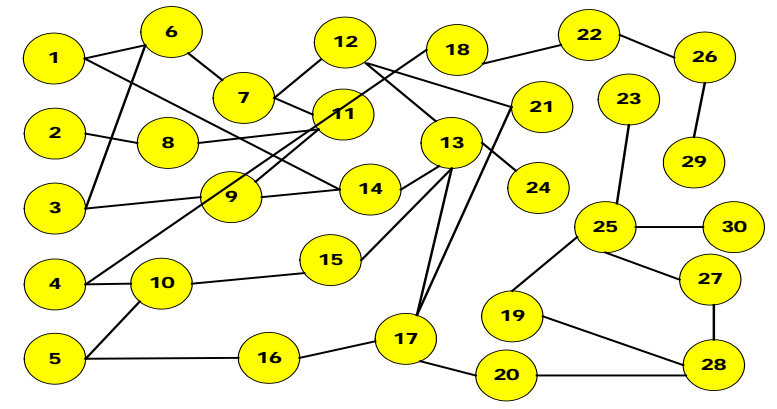

Fig. 2 Network Topology before Optimization

\section{Network topology analysis.}

Natural connectivity is used as the network topology invulnerability measurement to optimize the network topology. In the case of maintaining the same edge number, with the increase of the number of iterations, natural connectivity continues to increase and the optimal value is 1.4, which is obtained in the $397^{\text {th }}$ generation, and the natural connectivity is 1.4605 . Therefore, the combination of generic algorithm and cloud model can be used to solve the network topology optimization model, with fast algorithm convergence rate.

\section{Conclusion}

An important purpose of complex network research is to design good network topology. This paper established the network topology optimized model with natural connectivity as the objective, sought solution by combining with generic algorithm and cloud model and verified the model and method by simulation experiments.

\section{Acknowledgements}

This study was supported by Jiangsu Province Collaborative Innovation Center of Novel Software Technology and Industrialization. 


\section{References}

[1] Chvatal V. Tough graphs and Hamiltonian circuits [J]. Discrete Mathematics, 1973, 5 215-228.

[2] Barefoot C. A., Entringer R., Swart H. Vulnerability in graphs - a comparative survey [J]. Journal of Combinatorial Mathematics and Combinatorial Computing, 1987, 1 13-22.

[3] Cozzen M., Moazzami D., Stueckle S. The tenacity of a graph. Seventh International Conference on the Theory and Applications of Graphs [C]. New York: Wiley, 1995.

[4] Zhu Baoxuan. The algebraic connectivity of graphs with given matching number [J]. Graphs and Combinatorics,2012,29(10) 1-7.

[5] Hu Shenglong, Qi Liquan. Algebraic connectivity of an even uniform hypergraph [J]. Journal of Combinatorial Optimization, 2012,24(4) 564-579.

[6]ZHANG Kun,TAN Gexin,ZHANG Kechen, et al. Research review on invulnerability measure of complex network [J]. Computer era, 2010( 5) 4-7.

[7]WANG Xiaofan,LI Xiang,CHEN Guanrong. The theory and application of the complex network [M]. Beijing: Tsinghua University Press, 2006.

[8] WU Jun. Study on invulnerability of complex network topologies [D]. Changsha: National University of defense technology, 2008.

[9] Chen Jianan, GuoDawei, XuNaiping; Summary of theoretical studies on genetic algorithms [J]. Journal of Xidian University, 1998 , 25(3) 363-368.

[10] Yuan Lihua. Theoretical research on genetic algorithm based on evolution of species [M]. Nanjing University of Aeronautics and Astronautics, 2009.

[11] Sriniva M,Patnaik L M. Adaptive probabilities of crossover and mutation in genetic algorithm.IEEE Transactions on System, Manand Cybernetics, 1994, 24(4) 656-667.

[12] Wang Xiaoping, Cao Liming. Genetic algorithm theory, application and software implementation [M], Xi'an Jiaotong University Press, 2002.

[13] Li DY, Du Yi. Artificial intelligence with Uncertainty. Beijing:National Defense Industry Press, 2005.

[14] Li DY, Liu CY. Study on the universality of the normal cloud model [J]. Chinese Engineering Science, 2004,6(8) 28-34. 